\title{
Functional Outcomes of Unstable Traumatic Elbow Injuries: How and When to Fix Them
}

ISSN: 2576-8875

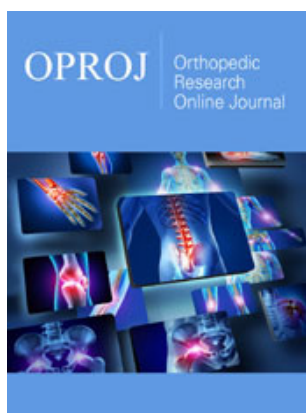

*Corresponding author: Mokhtar Abdul Azeem, Department of orthopedic trauma, Lecturer at Al-Azhar University Hospitals, Faculty of Medicine, Cairo, Egypt

Submission: 淟 September 28, 2020

Published: 此 October 07, 2020

Volume 7 - Issue 4

How to cite this article: Mokhtar Abdul Azeem, Awdhah Al-Samhan, Sager Hanna, Bader Almurad, Aliaa Khaja. Functional Outcomes of Unstable Traumatic Elbow Injuries: How and When to Fix Them. Ortho Res Online J. 7(4). OPROJ. 000666. 2020

DOI: 10.31031/OPROJ.2020.07.000666

Copyright@: Mokhtar Abdul Azeem, This article is distributed under the terms of the Creative Commons Attribution 4.0 International License, which permits unrestricted use and redistribution provided that the original author and source are credited.

\author{
Mokhtar Abdul Azeem ${ }^{1 *}$, Awdhah Al-Samhan¹, Sager Hanna1 , Bader Almurad ${ }^{2}$ \\ and Aliaa Khaja ${ }^{1}$ \\ ${ }^{1}$ Lecturer at Al-Azhar University Hospitals, Faculty of Medicine, Cairo, Egypt \\ ${ }^{2}$ Musculoskeletal Radiologist, Department of Radiology, Al-Razi Orthopedic Hospital, Kuwait
}

\begin{abstract}
Objectives: Complex elbow fractures have a predisposition for undesirable outcomes even with surgical management. The aim of this study was to evaluate the functional outcome of patients with traumatic unstable elbow Injuries that were managed surgically, as well as how to select the proper surgical approach and fixation guided by pre-operative planning. Analyzing their functional outcomes would help formulate future evidence-based guidelines on elbow terrible triad injury management in clinical practice.
\end{abstract}

Methods: All the surgical cases for Traumatic Unstable Elbow Injuries were collected from a single tertiary hospital in the country that deals exclusively with these types of injuries. The database included 19 patients that were treated between 2013 until 2018. All the patient agreed to participate in this study. They were asked to fill in the Mayo Elbow Performance Score (MEPS) at baseline first clinic after hospital discharge, and later at final follow up after completion of physiotherapy. Both functional outcomes and complications were correlated with clinical and radiological evaluation.

Results: A total of 19 cases of terrible triad of the elbowwereincluded in the studyanalysis. The study sample included 19 participants (31.6\% females and 68.4\% males). Results showed that MEPS and ROM significantly improved after the completion of follow up compared to the baseline. The median extension score decreased from 20 to $10(\mathrm{P}<0.05)$. The median flexion score increased from 122 to 140 ( $\mathrm{p}=0.001$ using Wilcoxon signed-rank test). The median supination score increased from 45 to $80(p<0.05)$ while the median pronation score increased from 42.5 to $70(\mathrm{p}=0.05)$. The median MEPS scores significantly increased from 60 to 85 ( $\mathrm{p}<0.001$ ). When analyzed as a categorical variable, results showed that the percentage of patients with good MEPS score increased from $21.1 \%$ to $68.4 \%$ (p< 0.001 using McNemar's test).

In terms of complications, 6 of the 19 patients needed a second surgical intervention with joint stiffness as the leading cause

Conclusion: Terrible triad injuries of the elbow are a result of high energy trauma. These types of injuries lead to unstable elbows that requires surgical intervention, extensive post-operative physiotherapy and a high possibility of reoperation is required. We conclude that good outcomes after this injury can be achieved by adequate preoperative planning, stable fixation, aggressive rehabilitation, regular outpatient follows-up and complication anticipation and management.

Keywords: Terrible triad;Elbow;Surgery;Joint;Complex fracture

\section{Introduction}

Traumatic Unstable Elbow Injuries were first described by Hotchkiss in 1996, and later defined as a dislocation of the elbow joint, combined with fractures of the radial head and ulnar coronoid process [1]. The definition extends to include the lateral collateral ligaments, the medial collateral ligaments and the anterior capsule. This is a high trauma injury caused by axial forces on a hyperextended elbow in supination [2]. This in turn will cause exaggerated elbow valgus that will ultimately lead to elbow instability [3].This condition accounts for $4 \%$ of all adult radial head fractures and $31 \%$ of elbow dislocations. The name associated with this injury reflects the bad outcomes that accompanied this injury despite surgical management $[4,5]$. This is why a good understanding of the injury mechanism, the relevant anatomy, and the factors associated with elbow instability can grant better functional outcomes and reduce the risk of complications.

Many patient-related outcome scores are used to evaluate elbow injuries and pathologies. Scores such as the Oxford Elbow Score, Liverpool Elbow Score and the Mayo Elbow 
Performance Score have proven to be useful and reliable tools tool in clinical practice [6-8]. The Mayo Elbow Performance Score (MEPS), however, has a higher validity rate and wider range of functional assessment. The normal range of motion of the elbow is approximately $0^{\circ}$ of extension and $140^{\circ}$ of flexion. A functional range of motion for activities of the daily living has been described to be of $30-130^{\circ}$, and the functional arc of throwing ranges from $20^{\circ}$ to $130^{\circ}$. The normal supination and pronation are both of approximately $80^{\circ}$ [5-7]. The desired functional arc of movement 50-50 degrees in two planes; flexion and extension (sagittal plane) and pronation and supination (coronal plane) . The MEPS was used to test the limitations of the elbow during activities of daily living (ADL). MEPS is a 4-part test where clinical information is rated based on a 100 points scale; <60-poor, 60-74 -fair, 75-89 - good, and 90-100 - excellent [7-10].

The objective of this study was to conduct a retrospective evaluation of the functional outcomes in 19 patients with Traumatic Unstable Elbow Injuries and how to apply a standardized approach to surgical planning in such injuries.

\section{Materials and Method}

The cases were collected form the medical records database of the only tertiary orthopedic hospital in the country. The data included 19 terrible triad cases of the elbow, from January 2013 to December 2018. These cases have been treated by an exclusive upper limb trauma service. The patients' files were retrieved, and the patients were contacted accordingly to re-visit the clinic for further evaluation. Ethical approval was first sought out and obtained from the Ministry of Health in the State of Kuwait (Reference code 2019/891). Written consent from each patient was also obtained. Patients were asked to visit outpatient clinic after discharge once a month for the first three months followed by once in 2-3 months later up until 24- months.

\section{Radiological evaluation}

We retrospectively analyzed data from their records, as well as accessing the hospital's PACS system to obtain and review radiological studies used for the patients. These were standard radiographs (Anterior-posterior and lateral views of the elbow), the CT scans of the affected elbows, as well as additional radiographs depending on each patient's injuries to assist in the pre-operative plan and in the duration of post-operative follow-up.

Radial head fractures were classified according to mason classification for radial head fractures [11]. Coronoid fractures were classified according to Regan and Morrey (for posterolateral and posteromedial instability) and O'Driscoll(for the anteromedial facet) classifications[5,12]. The radiographs were reviewed independently by two surgeons and where there was any doubt regarding the classification, the main Surgeon was asked for confirmation. Post-operative follow-up was done by the main surgeon, who determined whether there were any complications.

\section{Pre-operative planning and choosing the approach}

The first step in pre-operative planning is obtaining Anteroposterior and lateral radiographs of the elbow, as well as the involved bones above and below the elbow joint. Furthermore, a CT Scan of the Elbow joint with axial, sagittal, coronal, and 3-dimensional reconstruction views with volumetric measurement of the fracture fragments is done.

Volumetric sizing for both the radial head and the coronoid were done by multiplying the length, height and width together. These different cuts enable us to accurately classify fractures of the radial head, coronoid, and any additional fractures that might exist. The volumetric measurement is requested to determine the implant type and size for fixation. The choice of surgical approach was based on the algorithm in Figure 1, the choice of implant was based on the algorithm in Figure 2.

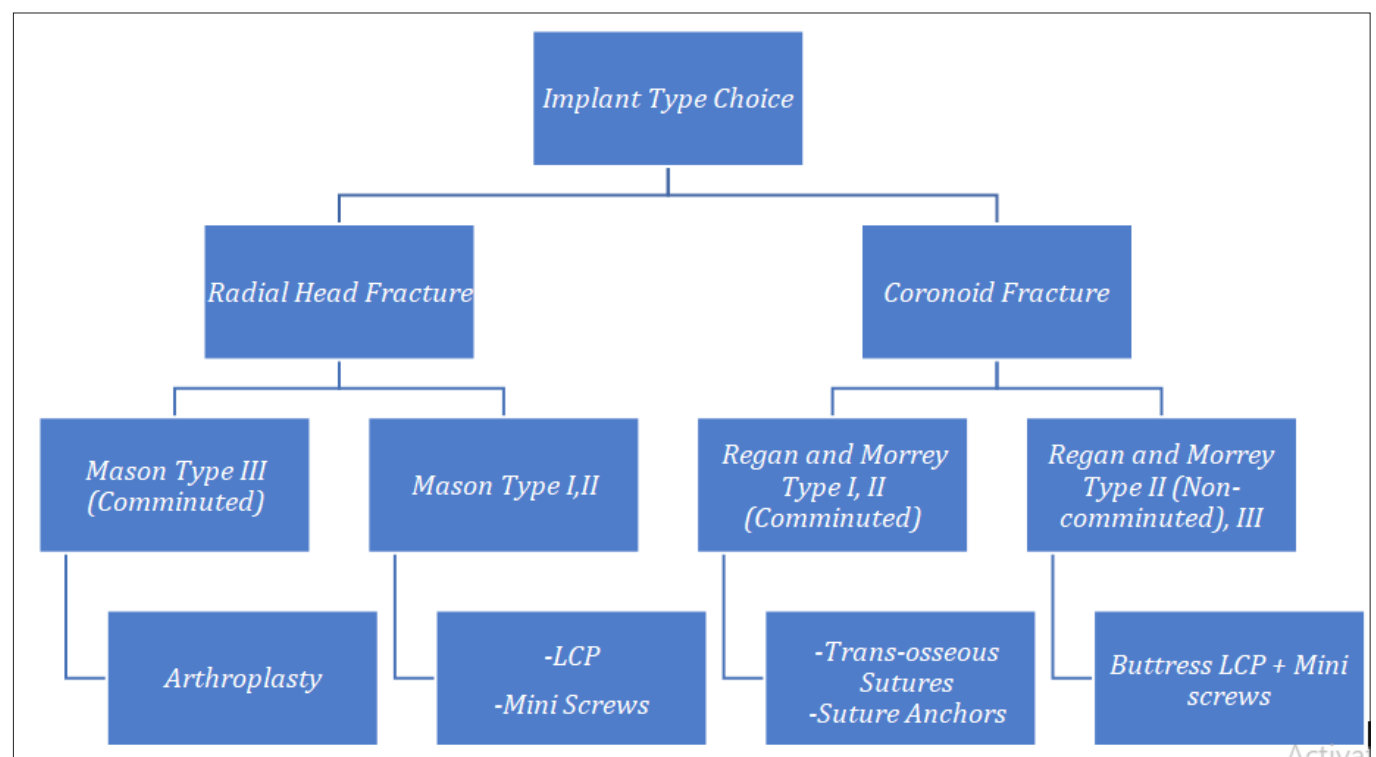

Figure 1: Algorithm used by authors to decide implant type. 


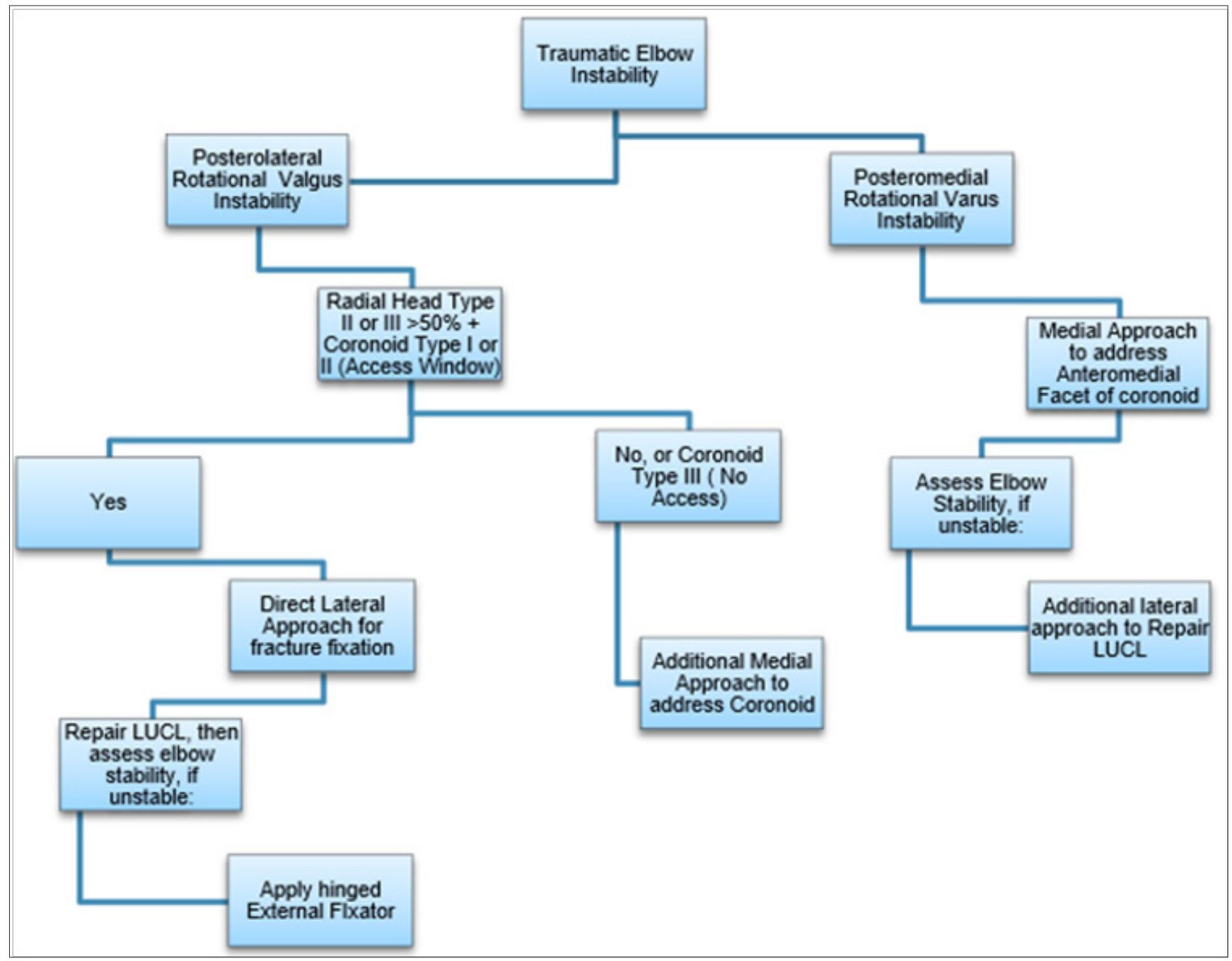

Figure 2: Algorithm for implant choice in unstable elbow injuries.

\section{Statistical analysis}

Statistical analysis was performed using R studio v 3.6.2. Demographic characteristics were summarized as counts and percentages while continuous variables were summarized using median and interquartile range (IQR) due to the non-parametric nature of the data. The percentages for the type of repair, complications, and associated injury was calculated from the total number of patients. Signed rank test was used to assess whether the mean ranks for the mayo score, flexion, extension, supination, and pronation were significantly different between baseline and follow up. Mayo score was classified as previously mentioned. McNemar's test was used to assess whether the distribution of Mayo score significantly changed at follow up compared to baseline. Signed rank and McNemar's test were used due to the paired nature of the data (each patient had two scores for each index).

\section{Results}

The study sample included 19 participants (31.6\% females and
$68.4 \%$ males). The median age of the included participants was 37 years (IQR 30.5; 47 years). Radial head fracture grades I, II, and III were found in $10.5 \%, 31.6 \%$, and $57.9 \%$ of the patients, respectively. Coronoidhead fracture grades I, II, and III were found in $52.6 \%$, $36.8 \%$, and $10.5 \%$ of the patients, respectively. MCL repair was performed in $21.1 \%$ of the patients while LCL and capsule repair were performed in all the patients. The median length of hospital stay was 8 days (IQR 6; 12 days). Cefazolin (1g q8 for 3 days) was the most commonly administered antibiotic. The mechanism of injury and occupation are shown in Table 1.

\section{Operative technique and rehabilitation protocol}

All patients were managed by the same surgical team. The same standard approach was used for all patients (Kocher or posterolateral approach). It was used to ensure exposure of the distal humerus as well as the radial head, radial neck, and biceps tuberosity. The medial approach was chosen in fractures that needed buttressing (Type II non-comminuted, type III and anteromedial facet fractures of the coronoid. 
Coronoid fractures were addressed first followed by the radial head fracture. Finally, the ligamentous and soft tissue injuries were addressed. All the cases underwent a Kocher approach, starting with radial head fixation or arthroplasty, followed by lateral collateral ligament repair. Then assessing for elbow instability; if the elbow was unstable elbows after lateral collateral ligament repair,

Table 1: Descriptive statistics for the study sample. a separate medial approachwas indicated to repair the medial collateral ligament as seen in Table 1. The approach was utilized to gain access and repair the anterior bundle of the medial collateral ligament. The last resort for patients with persistent instability was the usage of a hinged external fixator $(n=1)$.

\begin{tabular}{|c|c|c|}
\hline & [ALL] & $\mathbf{n}$ \\
\hline & $n=19$ & \\
\hline Gender: & & 19 \\
\hline Female & $6(31.6 \%)$ & \\
\hline Male & $13(68.4 \%)$ & \\
\hline Age & $37.0[30.5 ; 47.0]$ & 19 \\
\hline Occupation: & & 19 \\
\hline Bank Employee & $1(5.26 \%)$ & \\
\hline Branch Manager & $1(5.26 \%)$ & \\
\hline Builder & $1(5.26 \%)$ & \\
\hline Businessman & $1(5.26 \%)$ & \\
\hline Cleaner & $1(5.26 \%)$ & \\
\hline Engineer & $1(5.26 \%)$ & \\
\hline Factory worker & $1(5.26 \%)$ & \\
\hline Housemaid & $3(15.8 \%)$ & \\
\hline Housewife & $1(5.26 \%)$ & \\
\hline Manual Worker & $4(21.1 \%)$ & \\
\hline Sales Manager & $1(5.26 \%)$ & \\
\hline Student & $1(5.26 \%)$ & \\
\hline Teacher & $1(5.26 \%)$ & \\
\hline Unemployed & $1(5.26 \%)$ & \\
\hline Radial Head Fracture Classification: & & 19 \\
\hline I & $2(10.5 \%)$ & \\
\hline II & $6(31.6 \%)$ & \\
\hline III & $11(57.9 \%)$ & \\
\hline Coronoid Fracture Classification: & & 19 \\
\hline I & $10(52.6 \%)$ & \\
\hline II & $7(36.8 \%)$ & \\
\hline III & $2(10.5 \%)$ & \\
\hline Mechanism of Injury: & & 19 \\
\hline Fell at home & $1(5.26 \%)$ & \\
\hline Fell during sport & $2(10.5 \%)$ & \\
\hline Fell from Bicycle & $1(5.26 \%)$ & \\
\hline Fell in bathroom & $2(10.5 \%)$ & \\
\hline FFH & $7(36.8 \%)$ & \\
\hline FFSH & $1(5.26 \%)$ & \\
\hline MVC & $3(15.8 \%)$ & \\
\hline RTA & $1(5.26 \%)$ & \\
\hline Slipped down stairs & $1(5.26 \%)$ & \\
\hline
\end{tabular}




\begin{tabular}{|c|c|c|}
\hline Hospital Stay & $8.00[6.00 ; 12.0]$ & 19 \\
\hline Choice of Antibiotics: & $17(89.5 \%)$ & 19 \\
\hline Cefazolin 1g IV q8H for 3 days & $1(5.26 \%)$ & $1(5.26 \%)$ \\
\hline Dalacin C 300g IV for 7 days & $4(21.1 \%)$ & 19 \\
\hline Vancomycin 1g IV q8H for 3 days & $19(100 \%)$ & $19(100 \%)$ \\
\hline Soft tissue repair $\Delta$ & & \\
\hline MCL repair: & & \\
\hline LCL repair: & & \\
\hline Capsule repair: $\quad$ Continuous variables were summarized as median $[\mathrm{IQR}]$ & \\
\hline \multicolumn{2}{|c|}{$\Delta$ Percentage for each response was calculated from the total n } \\
\hline
\end{tabular}

One patient had fixation of coronoid fracture with a screw, while the rest had trans-osseous pull out sutures with nonabsorbable sutures as the choice of fracture fixation. One patient had a concomitant ipsilateral proximal ulna fracture which was addressed with open reduction and internal fixation with plate and screws.

Fixation options for the radial head were open reduction and internal fixation with plate and screws $(n=5)$, screws only $(n=10)$, and screw fixation augmented with a Kirschner wire $(n=1)$, and radial head replacement $(n=1)$. The lateral collateral ligament was repaired in all the cases using a trans-osseous suture. The medial collateral ligament was repaired also by using a trans-osseous suture $(n=4)$. A hinged external fixator was used when the elbow remained unstable even after medial collateral ligament repair during the intraoperative stability assessment $(n=1)$. All the patients were placed in an above elbow cast until time of suture removal (Range 0-14 days).

Associated injuries were treated on a case-to-case basis depending on the injury itself (Figure 3). All patients were referred to an outpatient physiotherapy program after discharge and had at least 12 sessions. The rehabilitation protocol is divided into 3 distinct phases with each phase being for 4 weeks. First phase involves active and active-assisted range of motion to prevent heterotopic ossification [13]. The second stage involves passive range of motion exercises to regain as manyrange of motion as possible. The third and final stage, the strengthening and stretching stage, helps patients to slowly return to their activities of daily living.

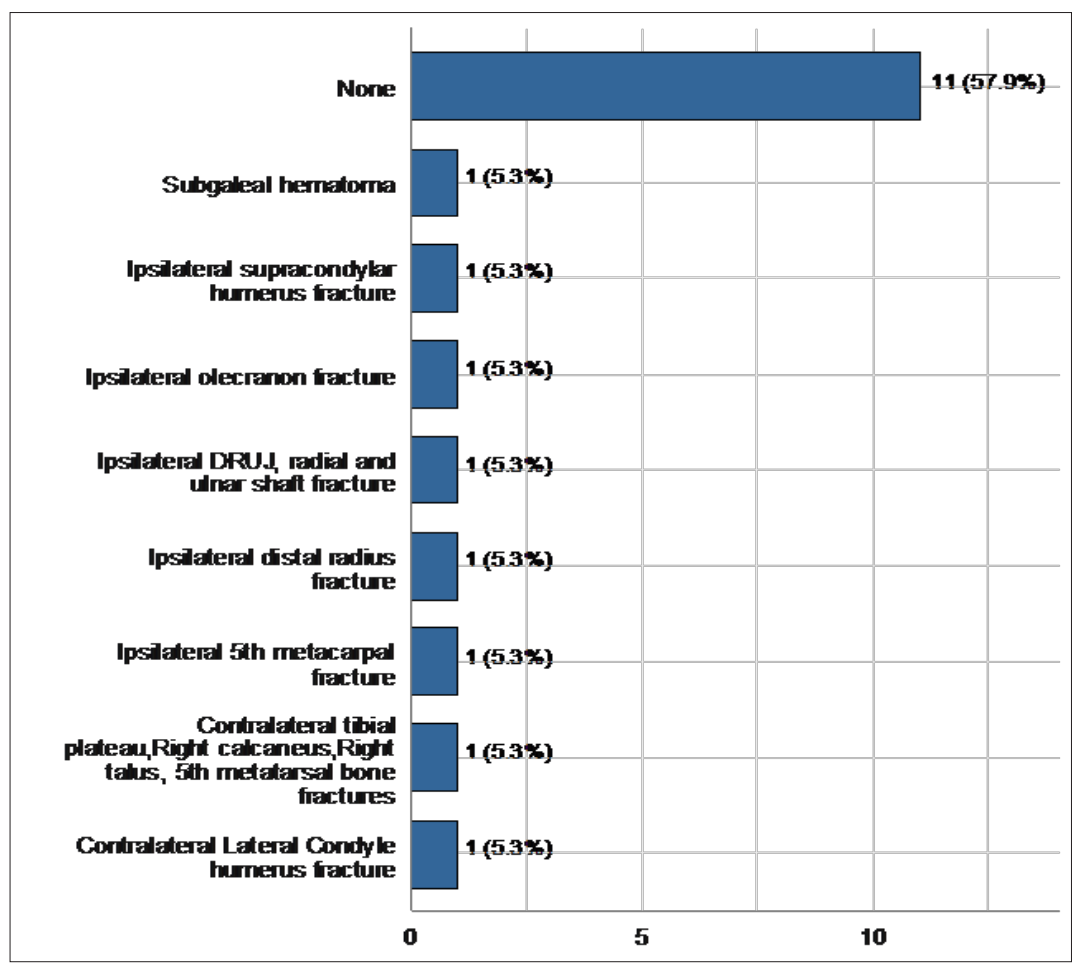

Figure 3: Associated injuries (Percentage for each injury was calculated from the total number). 


\section{Range of motion and MEPS}

Results showed that MEPS and ROM significantly improved after the completion of follow up compared to the baseline. The median extension score decreased from 20 to 10 ( $P<0.05)$. The median flexion score increased from 122 to 140 ( $p=0.001$ using Wilcoxon signed-rank test). The median supination score increased from 45 to $80(p<0.05)$ while the median pronation score increased from 42.5 to $70(p=0.05)$. The median MEPS scores significantly increased from 60 to $85(\mathrm{p}<0.001)$. When analyzed as a categorical variable, results showed that the percentage of patients with good MEPS score increased from 21.1\% to $68.4 \%$ ( $p<0.001$ using McNemar's test). Table 1, Figure 4\&Figure 5represent the change in MEPS and ROM at baseline compared to final follow up (Table 2).

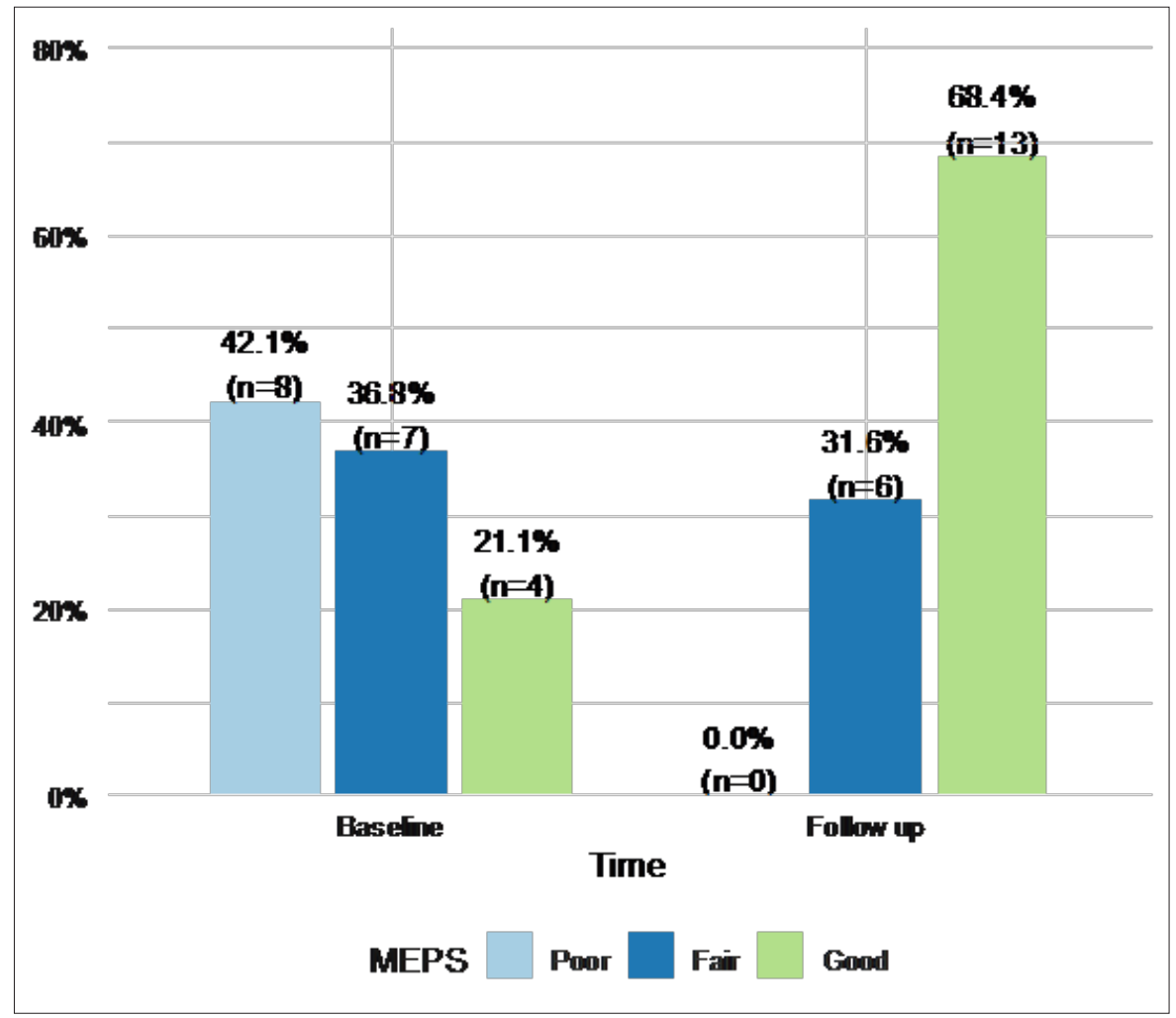

Figure 4:MEPS score categories at baseline and after completing follow up

Table 2: Change in ROM and MEPS measures.

\begin{tabular}{|c|c|c|c|}
\hline & Baseline & Follow up & $\mathbf{n}=\mathbf{1 9}$ \\
\hline & $\mathbf{n = 1 9}$ & $10.0[0.00 ; 20.0]$ & 0.03 \\
\hline Extension & $20.0[10.0 ; 30.0]$ & $140[132 ; 140]$ & 0.001 \\
\hline Flexion & $122[100 ; 132]$ & $80.0[47.5 ; 80.0]$ & 0.030 \\
\hline Supination & $45.0[25.0 ; 60.0]$ & $70.0[45.0 ; 80.0]$ & $\mathbf{0 . 0 5}$ \\
\hline Pronation & $42.5[20.0 ; 80.0]$ & $85.0[70.0 ; 85.0]$ & $<0.001$ \\
\hline MEPS mean & $60.0[50.0 ; 67.5]$ & & 0.001 \\
\hline MEPS score: & & $6(0.00 \%)$ & \\
\hline Poor & $7(36.8 \%)$ & $6(31.6 \%)$ & $13(68.4 \%)$ \\
\hline Fair & $4(21.1 \%)$ & & \\
\hline
\end{tabular}

Statistical analysis was performed using Signed-rank test for continuous variables and McNemar's test for categorical variables 


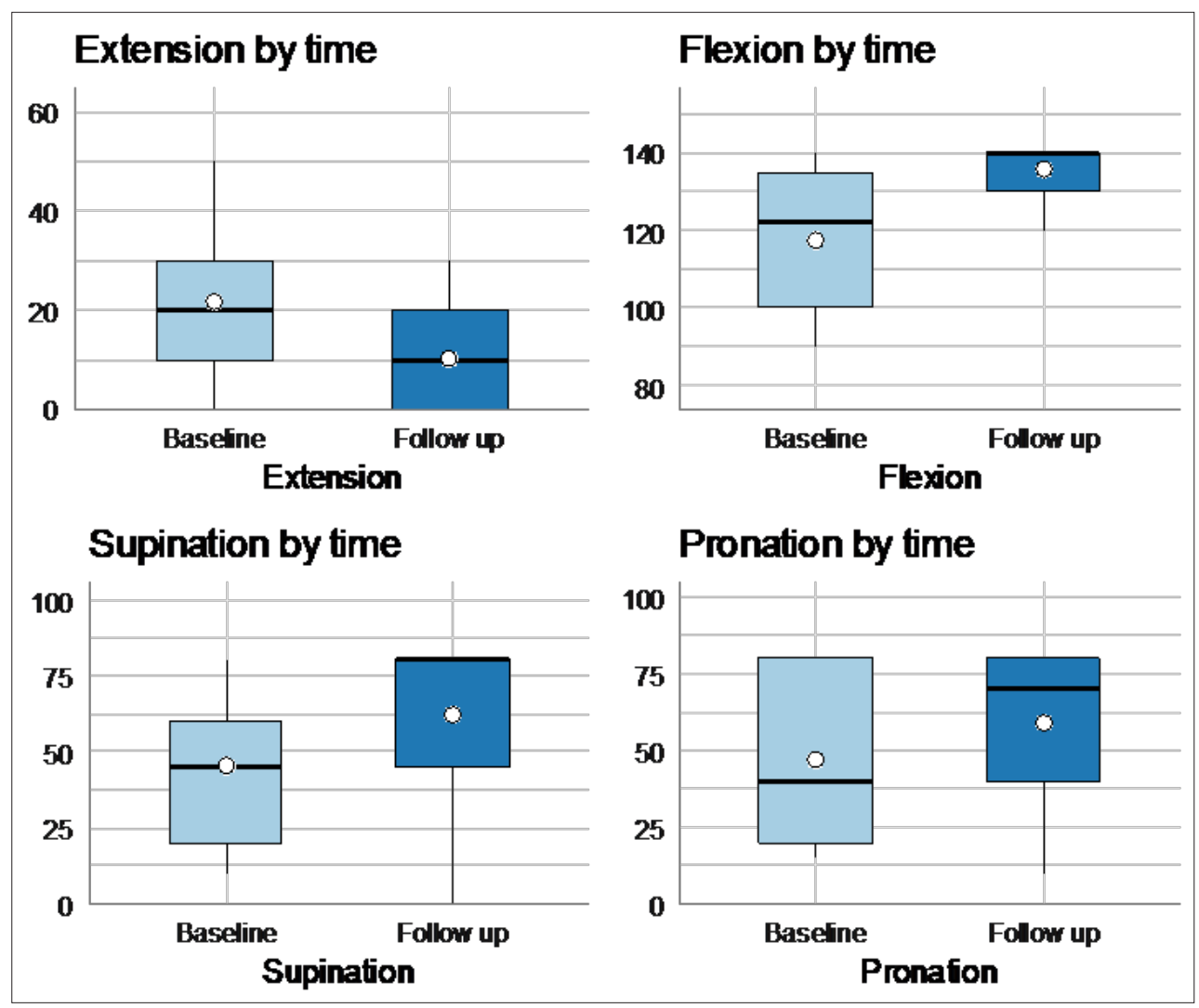

Figure 5:Change in $\mathrm{ROM}$ at baseline and after completing follow up.

\section{Complications}

From all the 19 patients included in the study, 3 were complication-free while 16 suffered at least one complication. Complications that we came across were limited range of motion in the joint without a significantly effected functional $\operatorname{arc}(n=11)$, Heterotopic ossification $(n=4)$ related to medial collateral ligament repair $(n=4)$, Ulnar nerve neuropraxia $(n=8)$ in which one case needed ulnar nerve exploration and hardware irritation $(n=1)$ (Figure 6).

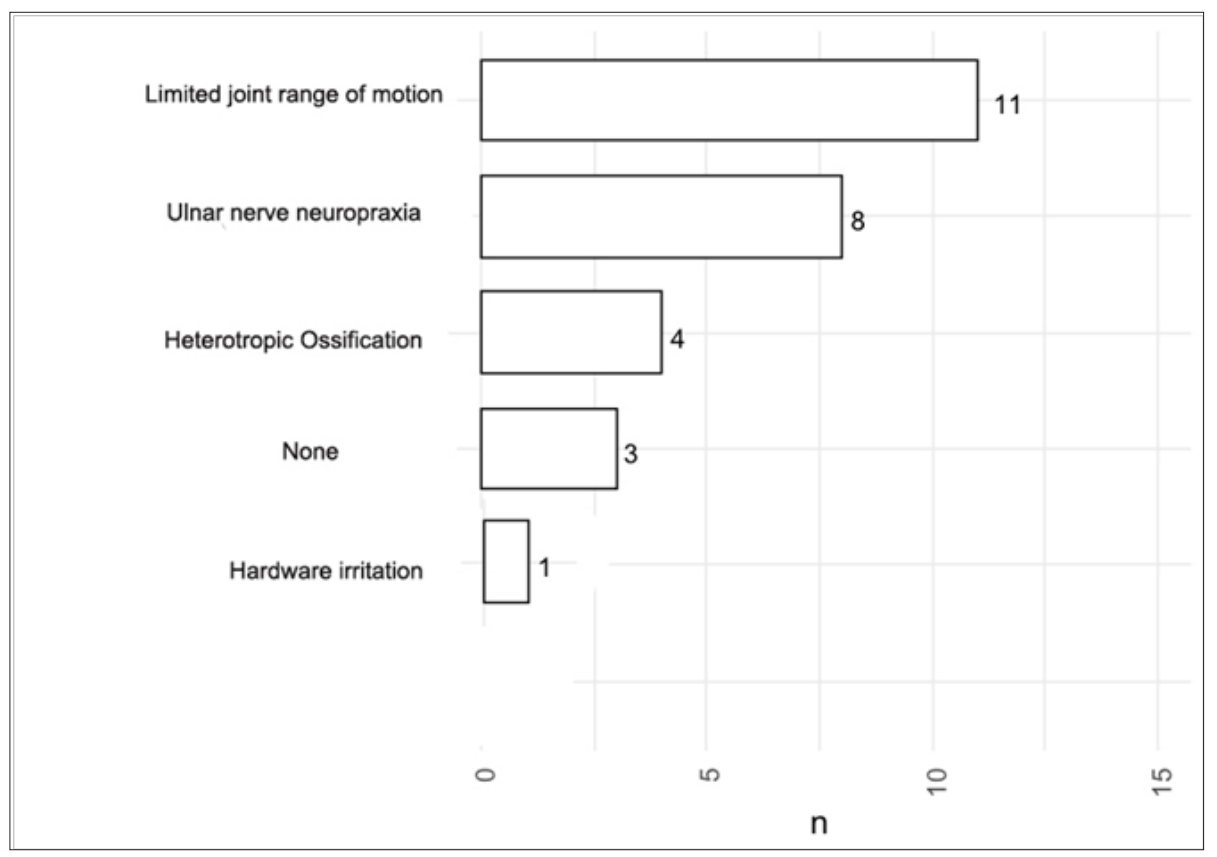

Figure 6: Complications experienced by the included patients (\% was calculated from total $\mathrm{n}$ ). 
Even though 16 had varying complications, only 4 patients ended up undergoing further operations beyond the first definitive surgery. These were Manipulation under anesthesia to assess persistent joint instability with possibility of re-applying a hinged Ex-fix $(n=1)$; heterotopic ossification excision and manipulation under anesthesia for joint stiffness $(n=1)$; ulnar nerve exploration $(n=1)$, manipulation under anesthesia for stiffness and removal of irritating hardware $(\mathrm{n}=1)$; ulnar nerve exploration and heterotopic ossification $(\mathrm{n}=1)$; and manipulation under anesthesia with ulnar nerve exploration and transposition $(\mathrm{n}=1)$.

\section{Discussion}

Terrible triad injuries of the elbow are uncommon yet are almost always accompanied by inadmissible complications [14]. There are many different surgical techniques and surgical approaches for each component of this triad. Each technique has proven to add some benefit to improve elbow stability or reduce associated complications [15-18]. No single surgical technique -to our knowledge- has been determined more superior than others [19].Planning to manage these types of injuries have to take into consideration tailored indications and patient needs [19]. Though one fact remains clear, that any non-surgical intervention will result in assertive disabilities to the joint [20-22]. The purpose of this current study was to examine the functional outcomes of unstable elbow injuries and their complications with surgical management and early rehabilitation. Algorithms for unstable elbow injuries were created to simplify the flow of operative decision making.We followed it for all the patients included in the study and achieved good results according to the MEPS. Compared with other similar studies, the strength of this paper is that there were no poor results amongst our cohorts [19-22].

Our study has achieved a similar result with the few other studies available in the literature [19,23-26]. This study has notably reported good to excellent results with adequate multi-dimensional surgical treatment. This is in keeping with other reports that used MEPS as their outcome measure as well [19,20,23]. Our cohorts had exhibited good results in return of elbow function to near normal (Figure 3). This study differs from others in that our participants did not report any poor MEPS cases. In terms of complications, our results are within the lower border of the normal range for elbow stiffness, heterotrophic ossification, and need for reoperation $[27,28]$. This too has been consistent with our initial hypothesis (Figure 4).

The methods used in managing the cases presented in this study followed a basic newly constructed algorithm, based on the latest evidence-based medicine presented in the literature regarding unstable elbow injuries. For the purpose of simplicity, the authors wish to base their discussion around coronoid fractures and their concomitant injuries in terrible triad injuries of the elbow. When the dislocation is stable and concentric, with non-displaced Regan-Morrey type I or II coronoid fracture or small anteromedial coronoid fracture, the literature suggest that these are indications for non-operative management [28]. These types of fractures should be immobilized at 90 degrees flexion for 14 days. Followed by active and assisted range of motion exercises [29,30].

Elbow instability or ulno-humeral joint subluxation generally benefits from surgical intervention.Coronoid fractures with valgus posterolateral rotational instability, or with varus posteromedial rotational instability or olecranon fracture-dislocation with or without radial heal fractures should be managed operatively to reduce the high risks of associated disabilities [31] (Figure 6).

Fixing the radial head in terrible triad injuries is straight forward in the literature recommendations. If the radial head is comminuted, the treatment is based on the size of the fragments $[31,32]$. If there are multiple large fragments that can be reduced and fixated internally then it is best to do so [33]. If the comminution of the radial head involves more than two thirds of the radial head, or if the fragments are irreducible, then in this case one has to consider arthroplasty reconstruction of the radial head [33,34].In both open reduction and internal fixation of the radial head versus arthroplasty, it is important to plan your surgery around the radial neck integrity [35].

It is worth noting that fixation with arthroscopic-assisted surgery in terrible triad injurie is a promising field, but it will require highly skilled surgeons with long-learning curves. Ligamentous repair on the other hand encompass various techniques and methods, each method has specific indications to ensure optimal results [3436]. No single technique is superior to treat the different types of injuries, therefore, choosing which method should be tailored as per individual case needs. After fixation of the bony components of terrible triad injuries and repair of the lateral collateral ligament, one must assess stability of the joint by performing a gravity stress test intraoperatively [37]. If the elbow remains unstable, repair of the medial collateral ligament is required or preferably applying a hinged external fixator. The decision should be influenced by the fact that studies have shown an increased risk of heterotopic ossification when repairing the medial collateral ligament $[37,38]$.

How one fixes terrible triad injuries with posterolateral rotational instability of the coronoid is through different approaches. The lateral approaches: Kaplan and Kocher approaches are useful in comminuted coronoid fracture Regan-Morrey types I-II with radial head fractures type II or II. These lateral approaches will provide adequate access. The current recommendation is to use an inside-out technique, starting with fixation of the coronoid followed by radial head fixation or reconstruction and ending with lateral collateral ligament repair [38,39].

The medial approaches such as the trans-flexor carpi ulnaris and the Hotchkiss over-the-top approaches can be used to gain better access if needed. Sequential or posterior approaches also provide additional windows of access. These approaches can be utilized in coronoid Regan-Morrey type I-II fractures that are not comminuted with radial head fractures of any type $[33,36,37]$. Access to the radial head fixation and lateral collateral ligament 
repair can be done via the lateral window. The coronoid fracture as well as the medial collateral ligament can be done through the medial window [33]. Fixation in this case starts from the medial structures and end with the lateral structures. Coronoid fractures Regan-Morrey types I-II which are comminuted can have the tip and capsule repaired using a trans-osseous suture. Regan-Morrey coronoid fractures types II-III can be treated with open reduction screws, a mini plate or Kirschner wires [35].

When posteromedial rotatory instability in involved in terrible triad fractures of the elbow, the injury is in the anteromedial coronoid facet with a lateral collateral injury [34-36]. When faced with this type of fracture utilizing medial and lateral windows is best. A buttress plate to fix this bony structure is sufficient. Posterior approaches can also be used when coronoid, olecranon and radial fractures are all present. This can be achieved by medially elevating the entire flexor pronator mass, using the olecranon as an osteotomy to be able to fix the coronoid with a retrograde screw [36].

Finally, the authors recommend using the algorithms provided in this study when faced with complex elbow injuries.Future comparative studies should be performed to further elucidate the optimal protocol for treating these injuries.

\section{Limitations}

The retrospective design of this study subjects the results to a number of observational and experience biases. However, it was necessary to study the effects of the protocols used on patient functional outcomes and associated complications. The notable small sample size has also been disadvantageous. However, despite the small sample size, complex injuries are relatively scarce in the study population. It would have been preferable to compare results with other centers, but this was not possible, since this is the only center in the country that deals with such injuries.

\section{Conclusion}

Complex elbow fractures traditionally had bad results, more specifically terrible triad fracture/dislocations. There is a high reoperation rate due to associated complications. However, the authors conclude that good outcomes can be achieved with surgical intervention.Managing these patients with a well-planned and well-staged surgery to insure anatomical restoration of the bony and ligamentous structures is vital. In addition, aggressive physical rehabilitation is equally important factor in the process, albeit resulting in better functional outcomes.

\section{References}

1. Chen S, Yu SY, Yan H, Cai JY, Ouyang Y, et al. (2015) The time point in surgical excision of heterotopic ossification of post-traumatic stiff elbow: recommendation for early excision followed by early exercise. J Shoulder Elb Surg 24(8): 1165-1171.

2. Desai MM, Sonone SV, Badve SA (2006) Terrible triad of the elbow: A case report of a new variant. J Postgrad Med 52(1): 43-44.

3. Cui H, Yu Y, He Y (2018) Management of elbow stiffness after postoperative treatment of terrible triad elbow injury: maintaining mobility and stability using a combined protocol. International Orthopaedics (SICOT) 42(3): 609-618.

4. Duckworth AD, Wickramasinghe NR, Clement ND, McQueen MM (2014) Radial head replacement for acute complex fractures: what are the rate and risks factors for revision or removal? Clin Orthop Relat Res 472(7): 2136-2143.

5. Laumonerie P, Ancelin D, Reina N, Tibbo ME, Kerezoudis P, et al. (2017) Causes for early and late surgical re-intervention after radial head arthroplasty. Int Orthop 41(7): 1435-1443.

6. Morrey BF, Askew LJ, Chao EY (1981) A biomechanical study of normal functional elbow motion. J Bone Joint Surg Am 63(6): 872-877.

7. Cusick MC, Bonnaig NS, Azar FM, Mauck BM, Smith RA, et al. (2014) Accuracy and reliability of the mayo elbow performance score. The Journal of Hand Surgery 39(6): 1146-1150.

8. Schneeberger AG, Kösters MC, Steens W (2014) Comparison of the subjective elbow value and the mayo elbow performance score. Journal of Shoulder and Elbow Surgery 23(3): 308-312.

9. Celik D (2015) Psychometric properties of the mayo elbow performance score. Rheumatol Int 35: 1015-1020.

10. Dawson J, Doll H, Boller I, Fitzpatrick R, Little C, et al. (2012) Specificity and responsiveness of patient-reported and clinician-rated outcome measures in the context of elbow surgery, comparing patients with and without rheumatoid arthritis. Orthop Traumatol Surg Res 98(6): 652658.

11. Dzugan SS, Savoie FH $3^{\text {rd }}$, Field LD, O’Brien MJ, You Z (2012) Acute radial ulno-humeral ligament injury in patients with chronic lateral epicondylitis: an observational report. J Shoulder Elbow Surg 21: 16511655.

12. Iannuzzi NP, Leopold SS (2012) In brief: The mason classification of radial head fractures. Clin Orthop Relat Res 470: 1799-1802.

13. Lindenhovius A, Karanicolas PJ, Bhandari M, van Dijk N, Ring D (2009) Interobserver reliability of coronoid fracture classification: two-dimensional versus three-dimensional computed tomography. The Journal of Hand Surgery 34(9): 1640-1646.

14. Schneider JC, Simko LC, Goldstein R, Shie VL, Chernack B, et al. (2017) Predicting heterotopic ossification early after burn injuries. Annals of Surgery 266(1): 179-184.

15. Thayer MK, Swenson AK, Hackett DJ, Hsu JE (2018) Classifications in brief: Regan-morrey classification of coronoid fractures. Clin Orthop Relat Res 476(7): 1540-1543.

16. Mellema JJ, Doornberg JN, Dyer GS, Ring D (2014) Distribution of coronoid fracture lines by specific patterns of traumatic elbow instability. J Hand Surg Am 39(10): 2041-2046.

17. O'Driscoll SW, Jupiter JB, Cohen MS, Ring D, McKee MD (2003) Difficult elbow fractures: pearls and pitfalls. Instr Course Lect 52: 113-134.

18. McKee MD, Pugh DM, Wild LM, Schemitsch EH, King GJ (2005) Standard surgical protocol to treat elbow dislocations with radial head and coronoid fractures. Surgical technique. J Bone Joint Surg Am 87(Suppl 1(Pt 1): 22-32.

19. Motisi M, Kurowicki J, Berglund DD, Triplet JJ, Disla S, et al. (2017) Trends in management of radial head and olecranon fractures. Open Orthop J 11: 239-247.

20. Pierrart, J, Bégué, T, Mansat, P (2015) Terrible triad of the elbow: treatment protocol and outcome in a series of eighteen cases. Injury 46(Suppl 1): 8-12.

21. Muñoz-Mahamud E, Estrada JA, Ballesteros JR, Combalia A, FernándezValencia JA (2018) Long-term functional outcomes of the terrible triad of the elbow. Int J Adv Jt Reconstr 5(1): 8-13. 
22. Najd Mazhar F, Jafari D, Mirzaei A (2017) Evaluation of functional outcome after nonsurgical management of terrible triad injuries of the elbow. Journal of Shoulder and Elbow Surgery 26(8): 1342-1347.

23. Jung SW, Kim DH, Kang SH, Eho YJ, Yang SW, et al. (2019) Risk factors that influence subsequent recurrent instability in terrible triad injury of the elbow. Journal of Orthopaedic Trauma 33(5): 250-255.

24. Kani KK, Chew FS (2019) Terrible triad injuries of the elbow. Emerg Radiol 26(3): 341-347.

25. Tangtiphaiboontana J, Agel J, Beingessner D, Hébert-Davies J (2020) Risk factors for the development of heterotopic ossification in operatively treated terrible triad injuries. Journal of Shoulder and Elbow Surgery 29(4): e146.

26. Domos P, Griffiths E, White A (2017) Outcomes following surgical management of complex terrible triad injuries of the elbow: a single surgeon case series. Shoulder \& Elbow 10(3): 216-222.

27. Zeiders GJ, Patel MK (2008) Management of unstable elbows following complex fracture-dislocations the 'Terrible Triad' injury. J Bone Joint Surg Am 90: 75-84.

28. Leigh WB, Ball CM (2012) Radial head reconstruction versus replacement in the treatment of terrible triad injuries of the elbow. J Shoulder Elbow Surg 21(10): 1336-1341.

29. Watters TS, Garrigues GE, Ring D, Ruch DS (2014) Fixation versus replacement of radial head in terrible triad: is there a difference in elbow stability and prognosis? Clin Orthop Relat Res 472: 2128-2135.

30. Chan K, MacDermid JC, Faber KJ (2014) Can we treat select terrible triad injuries nonoperatively? Clin Orthop Relat Res 472: 2092-2099.
31. Antoni M, Eichler D, Kempf JF, Clavert P (2019) Anterior capsule reattachment in terrible triad elbow injury with coronoid tip fracture. Orthopaedics \& Traumatology: Surgery \& Research 105(8): 1575-1583.

32. Kyriacou S, Gupta Y, Bains HK (2019) Radial head replacement versus reconstruction for the treatment of the terrible triad injury of the elbow: a systematic review and meta-analysis. Arch Orthop Trauma Surg 139(4): 507-517.

33. Yan M (2015) Radial head replacement or repair for the terrible triad of the elbow: which procedure is better? ANZ J Surg 85(9): 644-648.

34. Motisi M (2017) Trends in management of radial head and olecranon fractures. Open Orthop J 11: 239-247.

35. Chen HW, Liu GD, Wu LJ (2014) Complications of treating terrible triad injury of the elbow: a systematic review. PLoS One 9(5): e97476.

36. Giannicola G (2015) Terrible triad of the elbow: is it still a troublesome injury? Injury 46 Suppl 8: S68-S76.

37. Ring D, Horst TA (2015) Coronoid fractures. J Orthop Trauma 29(10): 437-440.

38. Iannuzzi NP, Paez AG, Parks BG, Murphy MS (2017) Fixation of reganmorrey type II coronoid fractures: A comparison of screws and suture lasso technique for resistance to displacement. The Journal of Hand Surgery 42(1): e11-e14.

39. Lee SH, Lim KH, Kim JW (2019) Case series of all-arthroscopic treatment for terrible triad of the elbow: Indications and clinical outcomes. Arthroscopy: The Journal of Arthroscopic \& Related Surgery. 